\title{
The Determination of The Homologous Purity of Higher Normal Alkanes up to Dohectane with Capillary Gas Chromatography
}

\author{
Yoshiko URABE* and Kanichiro TAKAMIZAwA \\ Department of Applied Science, Faculty of Engineering, Kyushu University, \\ Hakozaki, Higashi-ku, Fukuoka 812, Japan
}

(Received July 28, 1993)

\begin{abstract}
The applicability of high-temperature capillary gas chromatography was tested for higher members of normal alkanes up to dohectane. The samples used were synthesized and showed a high state of purity. The linear relationships were proved between the logarithm of the retention time and the number of carbon atoms in the molecule, $n$, for the chromatography at an isothermal operation. It was possible to detect the difference in $n$ by one for octacontane samples. The homologous impurity was proved to be quantitatively determined by the chromatography under an isothermal operation or under a programmed-temperature operation for mixtures of a limited range of $n$. The latter mode operation was desirable to detect higher components as sharp peaks. Even for dohectane the high-temperature capillary gas chromatography could be applied for the purpose of purity determination.

KEY WORDS Normal Alkanes / Homologous Impurity / Dohectane High-Temperature Capillary Gas Chromatography / Capillary Column / Isothermal Operation / Programmed-Temperature Operation / Phase Transition /
\end{abstract}

Recent development of capillary gas chromatography enabled us to operate at very high temperatures up to $450^{\circ} \mathrm{C}^{* 1}$ with durable capillary columns. A polyethylene sample (Polywax-1000), which consisted of components having a wide range of the number of carbon atoms in the molecule, $n$, were analyzed. ${ }^{1}$ The column used was $8 \mathrm{~m}$ in length with a $250 \mu \mathrm{m}$ inner diameter and it was covered with a $0.1 \mu \mathrm{m}$ silicone film for high temperature use. On the analysis the components of $n>100$ were detected as a peak, although the exact characterization of the components were not given. It seems that high-temperature capillary gas chromatography is a very powerful and convenient tool to determine the purity of long-chain com-

* To whom correspondence should be addressed.

*1 $0{ }^{\circ} \mathrm{C} \sim 273 \mathrm{~K}$. pounds, especially normal alkanes (abbr. $n$-alkanes). Previously, the purity of long normal carboxylic acid with up to $n=69$ was examined by mass spectroscopy, but the quantitative data of the purity was not given. ${ }^{2}$

We have studied the phase transition behavior of pure higher $n$-alkanes. The modifications just below melting are interesting in their nature of dynamic conformational disorders. ${ }^{3}$ These were initiated with a clear indication of solid-solid transition only for the samples of high purity on the homologue. ${ }^{4,5}$ The purity of lower $n$-alkanes was easily determined by capillary gas chromatography at relatively low temperatures. ${ }^{5}$ Our recent studies have focused on longer $n$-alkanes up to $n=80$ to 102 . Some peculiar thermal behavior 
of the high-temperature modifications of henpentacontane and dopentacontane was reported. ${ }^{6}$ We have to establish a reliable method for the determination of sample purity by using a high-temperature capillary gas chromatography. Here is the report on this subject.

\section{EXPERIMENTAL}

\section{Materials}

The samples examined were $n$-alkane, abbreviated as $\mathrm{C} n$, where $n$ is the number of the carbon atoms in the molecule. Highly pure samples were synthesized. The final chain extension for the even-numbered $\mathrm{C} n$ was done through Wurtz condensation, and that for the odd-numbered $\mathrm{C} n$ through a ketene dimerization reaction. ${ }^{5}$ Methyl esters of normal carboxylic acid were used as the starting substances after purification by molecular distillation. Their purity was more than $99.8 \%$. Dopentacontane C52, was derived from hexacosanoic acid (purchased from Sigma Chemical Co.) and dohexacontane, C62, from hentria contanoic acid (Sigma Chemical Co.). The esters were reduced to corresponding alcohols by using $\mathrm{NaBH}_{4}-\mathrm{AlCl}_{3}$ (3:1). This mild reduction was adopted to avoid a side reaction that led to a short-chain homologue. The alcohols were converted to corresponding normal alkyl iodides. For the synthesis of doheptacontane, C72, octacontane, C80, and dohectane, C102, two steps of a chain extension were needed, where icosanedioic acid (Okamura Oil Co.) and hexadecanoic acid (Sigma Chemical Co.), icosanoic acid (Sigma Chemical Co.), or hentriacontanoic acid, respectively, was used to synthesize longer $n$-alkyl iodides. The first step of the chain extension was Wurtz condensation between $\omega$-iodo, $\omega^{\prime}$-methoxyphenoxyicosane and $n$-alkyl iodide. The longer $n$-alkyl methoxyphenyl ether was easily separated from other components due to cross condensation, utilizing the difference in absorbability of end groups to the silica-gel col- umn. Henoctacontane, C81, was synthesized by two steps of a ketene dimerization reaction. Hentetracosanoic acid was first derived from icosanedioic acid and doicosanoic acid (Sigma Chemical Co.) then it was dimerized to 41-henoctacontanone, which was reduced to $\mathrm{C} 81$. The final purification of all the $n$ alkanes was performed by treatment with hot concentrated sulfuric acid and the subsequent elution, a heptane solution through the silica-gel column. It seemed that the purity of each sample was above $99.3 \%$ or more except for C81 and C102.

\section{Measurement}

A Shimadzu gas chromatograph, GC$14 \mathrm{AH}_{\mathrm{SC}}$, equipped with a data analyzer of C-R6A, was used. The instrument could be operated at an isothermal temperature mode up to $380^{\circ} \mathrm{C}$, however the highest temperature reached on a programmed-temperature mode was $450^{\circ} \mathrm{C}$. The metal capillary column used was CBN1-W15-020 (Nihon Chromato Works, Ltd.). It was $15 \mathrm{~m}$ in length with an inner diameter of $0.5 \mathrm{~mm}$ and it was coated with $0.2 \mu \mathrm{m}$ of polydimethylsiloxane film. Its. durability was good. ${ }^{7}$ We had used a fused silica column for the analysis of the lower members of $n$-alkanes. Although the maximum temperature on the heating run was about $400^{\circ} \mathrm{C}$, the column rapidly became fragile. This made systematic study difficult. Helium was used as the carrier gas, and its flow rate at ambient temperature was adjusted to $20 \mathrm{ml}$ $\min ^{-1}$. The volume injected was $1 \mu \mathrm{l}$ of a $0.1 \%$ toluene solution. Although a programmed-temperature split inlet system was used for the analysis of Polywax 1000, ${ }^{1}$ this inlet system was not suitable for our present analysis because these mixtures did not contain components of remarkably different homologues. The inlet system had an original split ratio of $1: 3$. This simple system was possible due to the use of the relatively wide bore on the column. FID detection was used and its temperature was set from $380^{\circ} \mathrm{C}$ to $440^{\circ} \mathrm{C}$. 


\section{RESULTS AND DISCUSSION}

At first, the relationships between retention time, $t$, and the number of carbon atoms, $n$, were examined at an isothermal condition. Figure 1 shows an example of the gas chromatogram for the mixture of equal weights of $\mathrm{C} 52, \mathrm{C} 62, \mathrm{C} 72$, and $\mathrm{C} 80$, eluted at $350^{\circ} \mathrm{C}$. Attention was paid to inject all components without any loss due to poor solubility of the higher components. Except for C52, the area for the components was approximately equal, as listed in Table I. A low value for C52 may be due to too fast of an elution compared with the others under this elution-temperature. In Figure 2, the retention, $t$, is plotted against $n$. The elution time for $\mathrm{C} 102$ was determined separately from the mixture of $\mathrm{C} 62, \mathrm{C} 72$, and C80. As is expected, the linear relationships between $\log t$, and $n$ hold. However, the data points for C52 at the high elution-temperatures deviate from the linear relationships. This may be due to the same reason as that stated above. These relationships are useful to assign to unknown impure components.

As seen in Figure 1, the elution peaks for the components of higher $n$ become very broad on the chromatogram at the isothermal condition. In order to detect clearly the components having higher $n$ as impurities, it is desirable to operate the chromatography with a programmed-temperature mode. The effectiveness of this analytical mode is indicated by the chromatogram in Figure 3, which was abserved for the same mixture as in Figure 1 with the operation from $270^{\circ} \mathrm{C}$ to $380^{\circ} \mathrm{C}$ at a heating rate of $2 \mathrm{~K} \mathrm{~min}^{-1}$. All the components

Table I. Quantitative analysis with the capillary gas chromatography for the mixture of equal weights of $\mathrm{C} 52, \mathrm{C} 62, \mathrm{C} 72$, and $\mathrm{C} 80$. The numeral is in $\%$ of weight

\begin{tabular}{lllll}
\hline Components & C52 & C62 & C72 & C80 \\
\hline $\begin{array}{c}\text { Constant } \\
\text { temp. }\end{array}$ & 22.4 & 25.2 & 26.5 & 25.7 \\
$\quad\left(350^{\circ} \mathrm{C}\right)$ & & & & \\
$\begin{array}{l}\text { Programming } \\
\text { rate }\end{array}$ & & & & \\
$2 \mathrm{~K} \mathrm{~min}^{-1}$ & 28.3 & 24.1 & 24.0 & 23.7 \\
$10 \mathrm{~K} \mathrm{~min}^{-1}$ & 24.9 & 25.1 & 25.6 & 24.2 \\
\hline Prepared & 25.06 & 25.01 & 24.49 & 24.96 \\
\hline
\end{tabular}

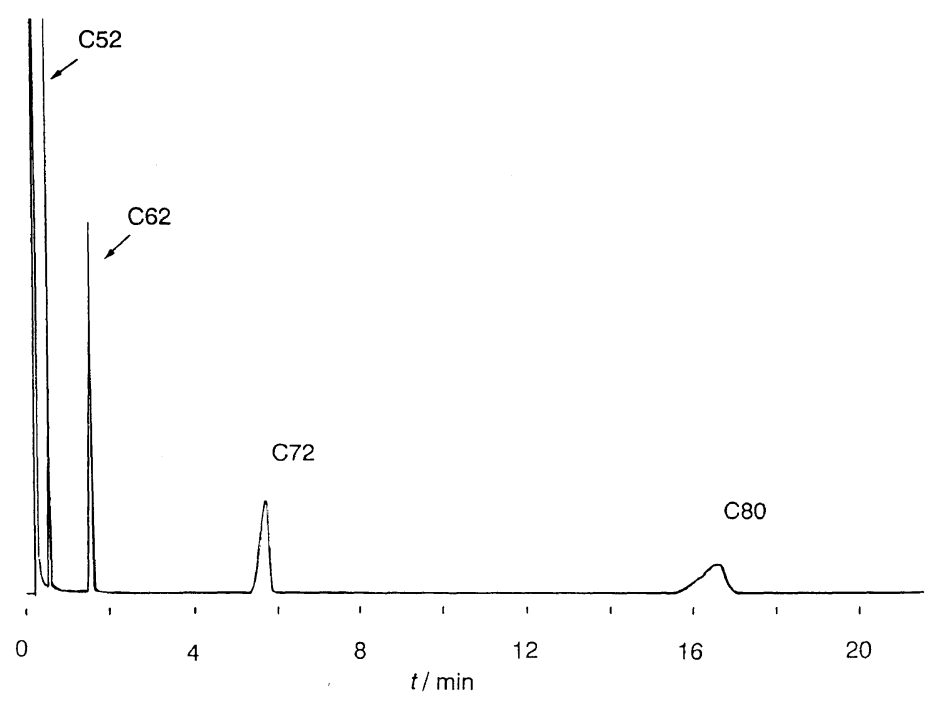

Figure 1. Capillary gas chromatogram of the $n$-alkane mixture of $\mathrm{C} 52, \mathrm{C} 62, \mathrm{C} 72$, and C80, eluted at $350^{\circ} \mathrm{C}$ through the column of CBN-1-W15-020. The flow rate of helium is $20 \mathrm{ml} \mathrm{min}{ }^{-1}$. The mixture consisted of the components of equal weight. 
give sharp peaks, and also the quantitativeness of the peak areas, as listed in Table I, is relatively good. The area of $\mathrm{C} 52$ is the highest contrary to the result of the isothermal operation. By magnifying the ordinate of the chromatogram the impure components could be discerned. A similar pattern was obtained up to a heating rate of $10 \mathrm{~K} \mathrm{~min}^{-1}$, but the base line was gradually deflected. Its quantita-

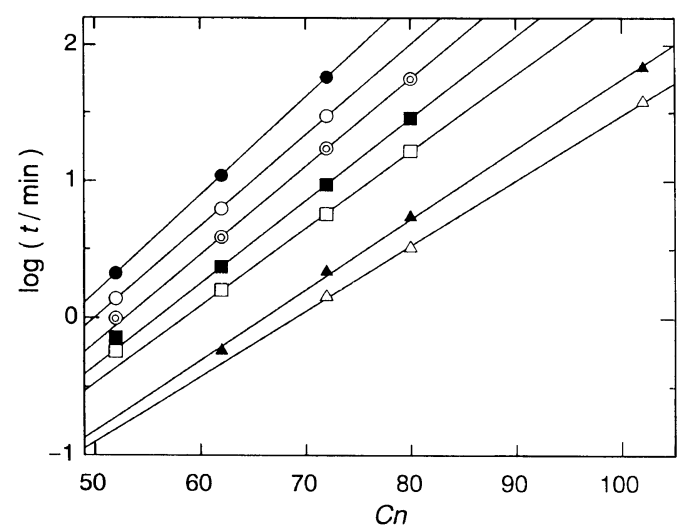

Figure 2. The linear relationships of the logarithm of the retention time, $t$, against the number of carbon atoms in the molecules, $n$, at various elution temperatures; $310^{\circ} \mathrm{C} ; \bigcirc, 320^{\circ} \mathrm{C} ; \odot, 330^{\circ} \mathrm{C} ; \square, 340^{\circ} \mathrm{C} ; \square, 350^{\circ} \mathrm{C}$, $370^{\circ} \mathrm{C} ; \triangle, 380^{\circ} \mathrm{C}$. tiveness is most satisfactory (Table I). It seems difficult to find the most suitable condition for the quantitative analysis of the mixtures having a wide range of $n$. The relationships between $\log t$ and $n$ for various programming rates are given in Figure 4. It may be possible to approximately assign $n$ of higher $n$-alkanes from these smooth curves despite of a lack of knowledge on the exact functional forms. The essential factors for the purity determination are the peak separation of the homologues and the quantitativeness of the peak areas. A severe test of the peak separation was done on the mixtures of C80 and C81. In Figure 5, a chromatogram of the $\mathrm{C} 80$ sample added with $0.5 \% \mathrm{C} 81$ is compared with that of $\mathrm{C} 80$ without the addition, because the $\mathrm{C} 80$ sample contains C81 of $0.08 \%$ and an unknown component of $0.76 \%$ at longer $t$ as an impurity. The chromatograms were obtained by an isothermal operation at $350^{\circ} \mathrm{C}$. In order to clearly show the very small peaks of the supplementary components, the scale of the ordinate of the chromatogram is magnified and then the top part of the C80 peak is cut.

It is concluded from the comparison that the homologues differing in $n$ by one are clearly

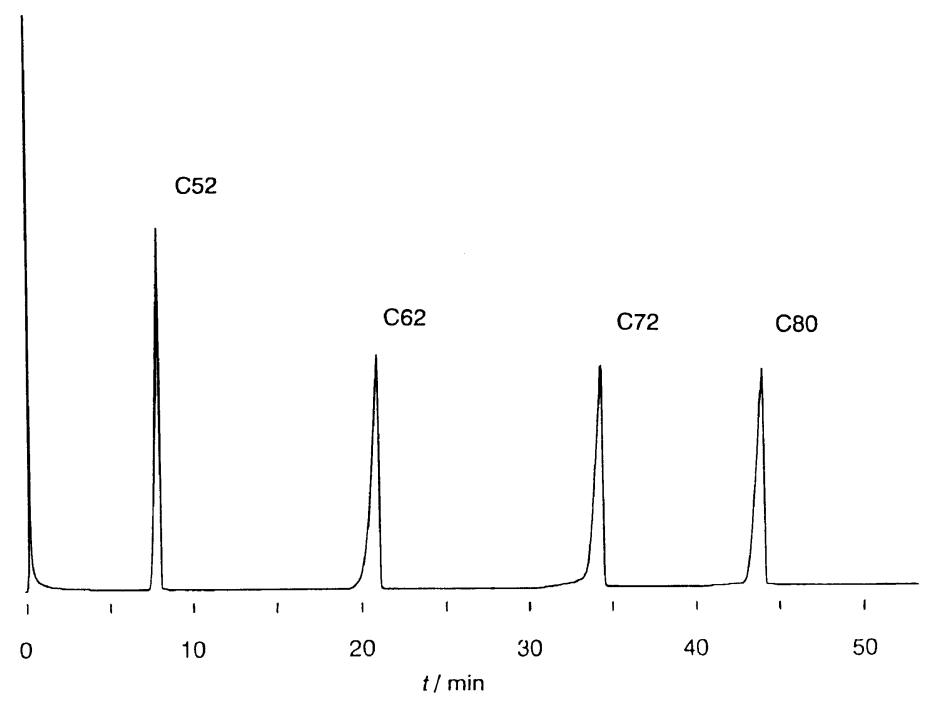

Figure 3. Capillary gas chromatograms of the same mixture as in Figure 1, with a programmedtemperature run at the rates of $2 \mathrm{~K} \mathrm{~min}^{-1}$ from $270^{\circ} \mathrm{C}$ to $380^{\circ} \mathrm{C}$. 
discerned for the higher $n$-alkanes of $n=80$.

The quantitative relationship over a range of low concentrations of $\mathrm{C} 72$ in the mixture with $\mathrm{C} 80$ was shown in Figure 6, in which the ordinate represents the observed concentration and the abscissa the prepared one. For weighing the prepared mixture, a Sartorius Supermicro Balance 4504 MP8, whose precision was $\pm 0.2 \mu \mathrm{g}$, was used. The analysis was

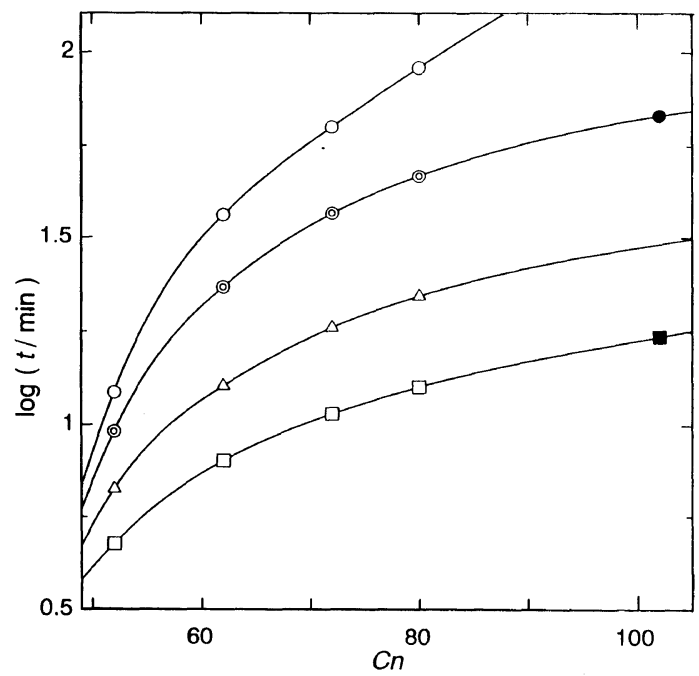

Figure 4. The relationships between the logarithm of $t$ and $n$ for the chromatography with a programmedtemperature mode at various rates of heating; $\bigcirc, 1$; $\odot$, $2 ; \triangle, 5 ; \square, 10 \mathrm{~K} \mathrm{~min}^{-1}$. Filled ones for $\mathrm{Cl} 102$ was separately measured.

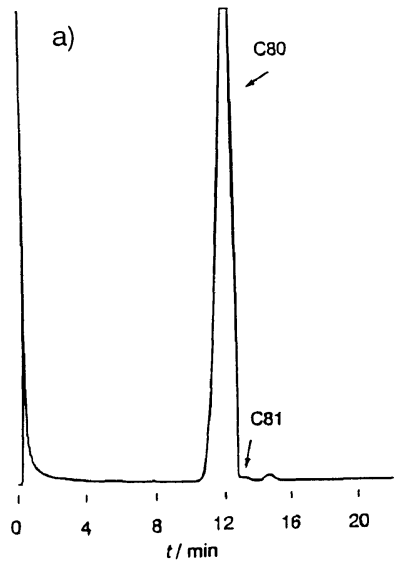

isothermally done at a column temperature of $345^{\circ} \mathrm{C}$. The quantiativeness is said to be satisfactory and the limit of detection may lie below about $0.1 \%$.

Recently, higher members of $n$-alkanes have become commercially available. This stimulates the studies of physical properties of $n$-alkane crystals. ${ }^{8} \mathrm{C} 60$ is the highest member asailable from Fluka AG and its nominal purity is indicated as $98 \%$. But, much attention has to be paid to the purity of the samples because the properties of the crystals are sensitively affected by the purity. In Figure 7 the comparison is made on two chromatograms: one for $\mathrm{C} 60$ purchased from Fluka and the other for C62 synthesized by us. It is seen that the commercial C60 contains lower and higher homologues. Its purity of $94.5 \%$, obtained by this analysis, is lower than the nominal value. On the other hand, our C62 showed a purity of $99.8 \%$. Much attention has to be paid to sample purity before discussion of the physical nature of the samples.

As an example, the DSC thermal behavior will be shown. The samples of our C52 $(99.8 \%$ pure) and C62 and Fluka C60 were crystallized from dilute heptane solutions and the dried mats of about $1.00 \mathrm{mg}$ were measured with a Rigaku DSC 8240B-TAS 100 system at a heating rate of $0.5 \mathrm{kmin}^{-1}$. The DSC curves

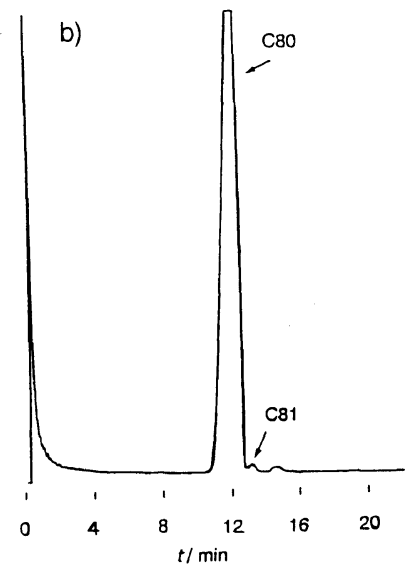

Figure 5. The comparison of the chromatograms for the sample of C80 (a) and for the sample added by $0.5 \%$ of $\mathrm{C} 81$ (b). Isothemal operation at $350^{\circ} \mathrm{C}$. 


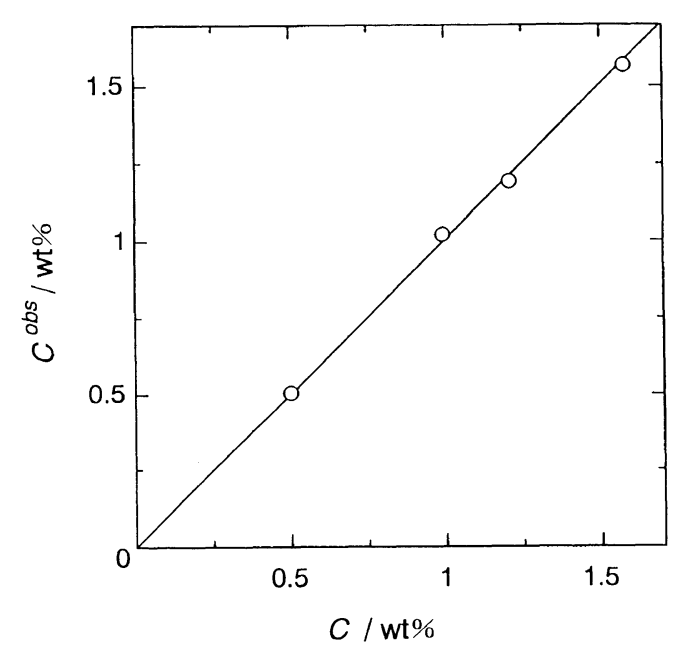

Figure 6. The relationship between the observed and the prepared concentration of $\mathrm{C} 72$ for the systems of $\mathrm{C} 72$ and C80. The column temperature was $345^{\circ} \mathrm{C}$.
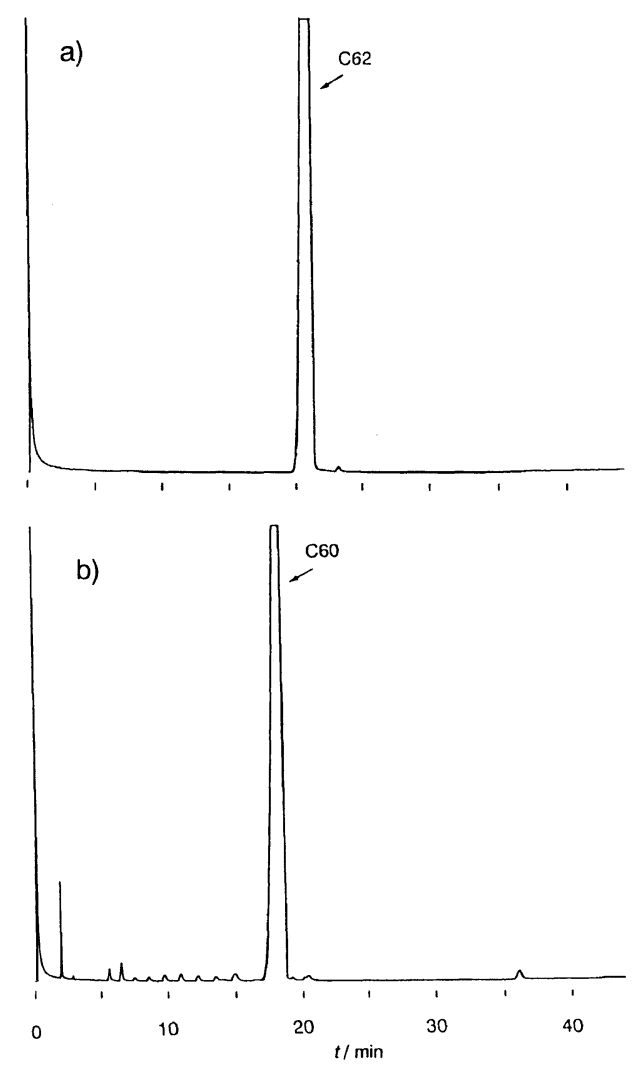

Figure 7. The comparison of the chromatograms between our C62 (a) and Fluka C60 (b). Programmedtemperature mode at a rate of $2 \mathrm{~K} \mathrm{~min}^{-1}$.

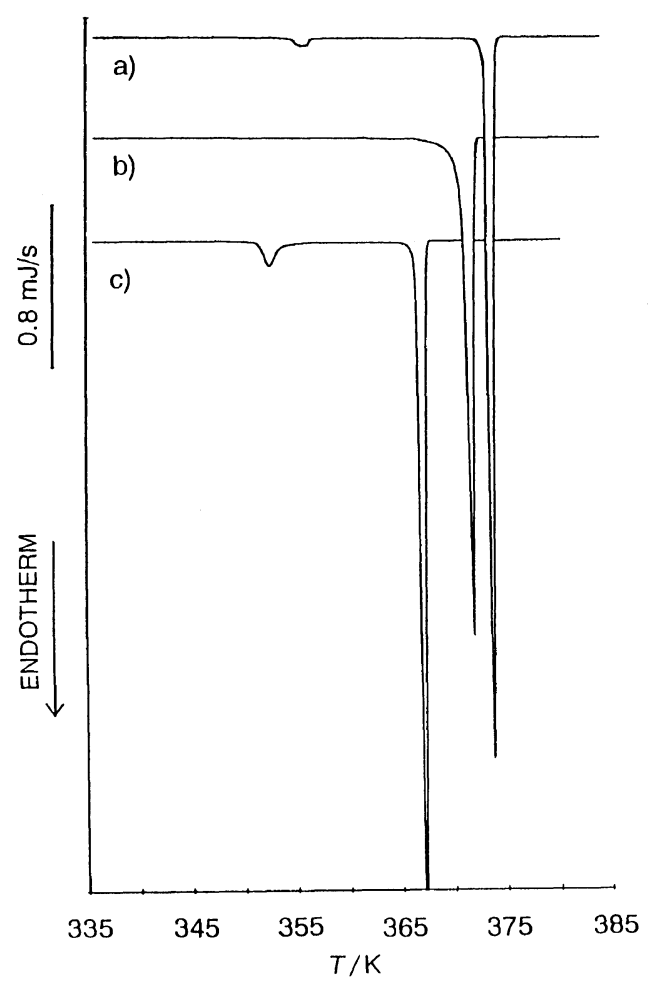

Figure 8. DSC curves for the samples crystallized from a dilute heptane solution: (a) C62, (b) Fluka C60, and (c) C52. Heating rate of $0.5 \mathrm{~K} \mathrm{~min}^{-1}$, sample weight of about $1.00 \mathrm{mg}$.

are drawn in Figure 8, in which the crystals of pure C52 and C62 clearly indicate small transition peaks before melting, as aforementioned. On the other hand, the C60 crystal only shows broad melting. It is a general fact that the crystals of higher $n$-alkanes transform from stable crystals at room temperature to the high temperature modification indicating the transition peak, as seen in Figure $8 .^{4,5,9}$ This fact has been missed when the purity of samples was not enough, as mentioned above. This is typically shown for the present C60 sample. The difference in the thermal behavior reflects the difference of the crystal structure at room temperature. Whereas the crystals of C52 and C62 had the monoclinic forms, the crystal of C60 was assigned to be orthorhombic. This structural difference may also hve resulted from 


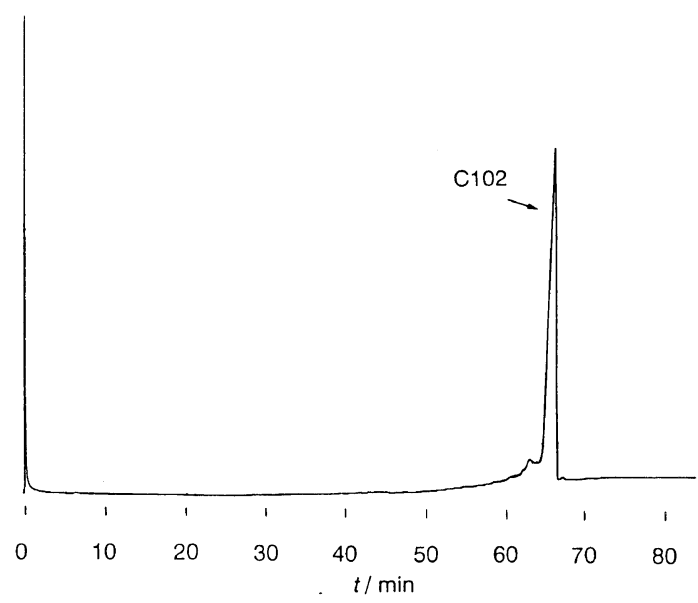

Figure 9. The chromatogram for the $\mathrm{C} 102$ sample observed at the heating rate of $2 \mathrm{~K} \mathrm{~min}^{-1}$ from $270^{\circ} \mathrm{C}$ to $440^{\circ} \mathrm{C}$.

the effect of the purity.

At last, the chromatogram of $\mathrm{C} 102$ is given in Figure 9. It was obtained by the programmed-temperature operation from $270^{\circ} \mathrm{C}$ to $440^{\circ} \mathrm{C}$ at a rate of $2 \mathrm{~K} \mathrm{~min}^{-1}$. Although the peak is relatively broad and the retention time is long, the small peak at a shorter $t$ may be ascribed to C96, but the substance appearing at the longer $t$ is unknown. It seems to be fairly stable because it remained after the treatment of hot sulfuric acid. The chromatogram suggests that an upper limit of the number of carbon atoms for $n$-alkanes to be analyzed by the capillary gas chromatography seems to locate at least a about 120 .

\section{CONCLUSION}

The present study shows that the high-tem- perature capillary gas chromatography is a very convenient and powerful means to analyze the sample purity of long-chain $n$-alkanes and also chain compounds. Up to the present, impurity within the sample of the $n$-alkane of $n=102$ could be detected with enough sensitivity.

Acknowledgment. This work was supported in part by Gran-in-Aid for Development Scientific Research (No. 03555191) from the Ministry of Education, Science, and Culture of Japan. The authors express appreciation to Prof. Yoshihiro Ogawa of Kumamoto University for the supply of henoctacontane sample and to Messers. Satoshi Tanaka and Seiji Tsuru for their assistance in the synthesis of $\mathrm{C} 80$ and $\mathrm{C} 102$.

\section{REFERENCES}

1. J. V. Hinshaw, Jr., J. Chromatogr. Sci., 25, 49 (1987).

2. A. Raal, G. Stallberg, and E. Stenhagen, Chem. Scrip., 3, 125 (1973).

3. B. Wunderlic, M. Moller, J. Grebowicz, and H. Baur, Adv. Polym. Sci., 87, 1 (1988).

4. K. Takamizawa, Y. Nagao, and Y. Urabe, Polym. J., 19, 981 (1987).

5. K. Takamizawa, T. Sonoda, and Y. Urabe, Kyushu Daigaku Sogorikogaku Kenkyuka Hokoku (Eng. Sci. Rep., Kyushu Univ.), 10, 363 (1989) (in Japanese).

6. Y. Urabe and K. Takamizawa, Chem. Lett., 2315 (1992).

7. Y. Takayama, T. Takeichi, and S. Kawai, J. High Res. Chromatogr. Commun., 11, 732 (1988).

8. Y. Kim, H. L. Strauss, and R. G. Synder, J. Phys. Chem., 93, 7520 (1989).

9. M. Maroncelli, S. P. Qi, H. L. Strauss, and R. G. Synder, J. Am. Chem. Soc., 104, 6237 (1982). 